\title{
Performance characterization and analysis for Hadoop K-means iteration
}

\author{
Joseph Issa
}

\begin{abstract}
The rapid growth in the demand for cloud computing data presents a performance challenge for both software and hardware architects. It is important to analyze and characterize the data processing performance for a given cloud cluster and to evaluate the performance bottlenecks in a cloud cluster that contribute to higher or lower computing processing time. In this paper, we implement a detailed performance analysis and characterization for Hadoop K-means iterations by scaling different processor micro-architecture parameters and comparing performance using Intel and AMD processors. This leads to the analysis of the underlying hardware in a cloud cluster servers to enable optimization of software and hardware to achieve maximum performance possible. We also propose a performance estimation model that estimates performance for Hadoop K-means iterations by modeling different processor micro-architecture parameters. The model is verified to predict performance with less than $5 \%$ error margin relative to a measured baseline.
\end{abstract}

Keywords: Performance prediction, Performance analysis, Hadoop K-means Iterations

\section{Introduction}

Given the rapid growth in the demand of cloud computing $[1,2]$ and cloud data, there is an increasing demand in storing, processing and a retrieving large amount of data in a cloud cluster. The data can be either stored to a cloud network such as scientific data (i.e. Climate modeling, Fusion, Bioinformatics...etc) or use the cloud network for data-intensive tasks such as collecting experimental data, dumping data on parallel storage systems, run large scale simulations...etc. Cloud computing is an emerging technology used to deliver different types of resources known as services over the internet. Cluster computing [3-7] is a set of stand-alone computers connected together to form a single computing resource $[8,9]$. This improves the performance and availability of a cloud cluster as compared to a single computer.

Hadoop was introduced as a solution to handle processing, storing and retrieving Big Data in a cloud environment which usually runs on a cluster of commodity machines. This cluster is composed of a master and slave nodes that process and compute data in parallel. It is important for processor architects to understand what

Correspondence: joseph_issa@yahoo.com

Department of Electrical and Computer Engineering, Notre Dame University, Zouk Mosbeh, Lebanon

(C) 2016 Issa. Open Access This article is distributed under the terms of the Creative Commons Attribution 4.0 International License (http://creativecommons.org/licenses/by/4.0/), which permits unrestricted use, distribution, and reproduction in any medium, provided you give appropriate credit to the original author(s) and the source, provide a link to the Creative Commons license, and indicate if changes were made. processor micro-architecture parameters contribute to higher or lower performance. It is also important for benchmark developers to optimize the benchmark software for a given hardware to achieve maximum performance possible. Hadoop is an open-source framework with two main components: MapReduce [10], and Hadoop Distributed File System (HDFS). HDFS is the primary storage for Hadoop; it is highly reliable and uses sockets for communications and is used for distributed storage [11, 12]. One important feature of HDFS is the partitioning of data and computation using thousands of hosts, and the execution of application computations in parallel in a way it is close to their data [13-16]. Hadoop cluster scales with computation and storage capacity by adding more servers. For example, Yahoo Hadoop cluster uses 40,000 servers and stores 40 PetaBytes of application data. Hadoop HDFS is used for data protection and reliability by replicating the file content across multiple DataNodes. This replication increases the probability for locating computation near the needed data.

The MapReduce $[17,10]$ framework is used for parallel processing. MapReduce and HDFS are co-designed, co-developed and co-deployed. What this means is that we have a single set of servers where MapReduce and HDFS are deployed so there is no separate set of servers

\section{Springer}


for HDFS to store data and a separate set of servers for processing data. One important aspect of MapReduce is that it's capability of moving compute to data (DataNode on which the data is located) and not the other way around. MapReduce knows where the data is placed in a cluster by working closely with HDFS. MapReduce consists of two main components, the JobTracker, and the TaskTracker. The JobTracker is the master and it is responsible for resource management such as tracking which nodes are up or down and how to deal with node failures. The TaskTracker is the slave, it gets direction from the JobTracker to run the tasks and report any failures and scheduling tasks.

Hadoop framework consists of several microbenchmarks developed using MapReduce algorithm, in other words, it is a framework used to process large data sets in a distributed computing environment. The resource utilization for the benchmarks is categorized as IO-bound, CPU-bound, or in between. Table 1 summarizes the system resource utilization for each workload.

In this paper, we present a detailed performance characterization for Hadoop K-means iterations using different processor configurations. We also propose a performance projection model that projects and model performance by changing different processor architecture parameters such as the number of cores/threads, memory bandwidth, memory size, cycles-per-instruction (CPI) and memory latency $[18,19]$. The remainder of this paper is organized as follows: In "Hadoop K-means Overview" section, we start with an overview of Hadoop K-means and Mahout K-means implementations. In "Related Work" section, compare our work to other published work of the same topic. In "Performance Characterization using Intel Xeon Based Platform" section we present a detailed performance characterization of Hadoop K-means for different key processor architecture parameters using Intel Xeon processor. In "Performance Characterization using AMD Interlagos Platform" section, we present a detailed performance analysis and characterization for Hadoop Kmeans using AMD Interlagos processor by analyzing the

Table 1 Workloads based on Hadoop framework: System Resource Utilization

\begin{tabular}{ll}
\hline Workloads & System Resource Utilization \\
\hline WordSort & $\begin{array}{l}\text { Sort Phase: IO-bound in the Reduce Phase: } \\
\text { Communication-bound. }\end{array}$ \\
Word Count & CPU-bound \\
TeraSort & Map Stage: CPU-Bound \\
& Reduce stage: IO-bound \\
Nutchlndexing & $\begin{array}{l}\text { IO-bound with high CPU utilizations in the map stage. } \\
\text { This workload is mainly used for web searching. }\end{array}$ \\
& $\begin{array}{l}\text { CPU-bound in the iteration, IO-bound in the clustering. } \\
\text { K-means }\end{array}$ \\
& It is used for machine learning and data mining.
\end{tabular}

performance sensitivity to key processor architecture parameters. In "Performance Projection Model"' section we propose a performance projection model that projects processor performance and total runtime and finally we conclude and discuss future work.

\section{Hadoop K-means overview}

Hadoop is designed as a framework for processing (storing and appending) multi Petabytes of data sets in a distributed computing cluster systems. There are several components of Hadoop architecture, the first component is known as the NameNode which is responsible for storing the file system namespace. The second component of Hadoop architecture is the DataNodes which is responsible for storing blocks and hosting MapReduce computation. The JobTracker component is responsible for tracking jobs; also it is responsible for detecting any failures. All applications in Hadoop are based on MapReduce which was introduced by Google. MapReduce means that a given application can be broken down into smaller blocks that can run on any node. The application can run on systems with thousands on nodes to achieve better performance. Hadoop is a framework which consists of several microbenchmarks. Some of these benchmarks are Sort, Word Count, TeraSort, K-means, and NutchIndexing. The file system in Hadoop is organized in a way that maps all the local disks in a cluster into a single file system hierarchy known as HDFS. Hadoop K-means is basically used for machine learning as well as data mining. It is divided into two main phases, the first phase is the iteration phase and the second phase is the clustering phase. In the iteration phase, the performance is a CPU-bound, which means the performance will increase if there is an increase in processing power such as an increase in the number of cores. In the clustering phase, the performance is IO-bound which means that the performance is limited and bounded by IO communication within a cluster. Clustering is a technique used to identify groups (clusters) within the input observation in such a way that the objects within each group will have high similarities and fewer similarities between other groups or clusters. The similarities metric in clustering algorithm uses distance measured only, similarities by correlation is not used in the clustering algorithm. K-means clustering generates a specific number of disjoint (non-hierarchal) clusters. The K-means method is numerical, unsupervised and iterative. $\mathrm{K}$ stands for $\mathrm{K}$ number of clusters and must be manually supplied by the user based on the input data.

Hadoop K-means version 2.7.0 is a clustering algorithm in which the input is a set of data points such as $\mathrm{K}$ with a set of points $\mathrm{X} 1, \mathrm{X} 2 \ldots . . \mathrm{Xn}$. The variable $\mathrm{K}$ refers to how many clusters it needs to find. The algorithm 
starts by placing $\mathrm{K}$ centroids in a random location such as $\mathrm{C} 1, \mathrm{C} 2 \ldots \mathrm{Ck}$. The algorithm will then repeat by executing the following steps below until convergence:

For each point $\mathrm{X}$, find the nearest centroid $\mathrm{C}$. This is done by computing the distance between $\mathrm{Xi}$ and $\mathrm{Cj}$ for every cluster centroid, then it pick the cluster J with minimum distance. It will then assign the point $\mathrm{X}$ to the cluster of the nearest centroid. For each cluster $J=1 \ldots . . . \mathrm{K}$, the algorithm will take the distance for every point in the selected cluster and average out the distance by using the following relation: $\mathbf{C}(\mathbf{a})=\frac{1}{n} \sum_{x i}^{\mathbf{c j}} \mathbf{X}(\mathbf{a})$ for $\mathbf{a}=1 \ldots \mathbf{d}$, where $C(a)$ is the new centroid for cluster J. The algorithm keeps running them one after the other until none of the cluster assignment changes, so no points change cluster membership. At this point, the algorithm has converged so it stops there. The complexity of the Hadoop K-means iteration algorithm is given as a factor of:

$$
\text { O(\#iterations } \times \# \text { clusters } \times \# \text { instances } \times \# \text { dimensions })
$$

The number of iterations is determined by how many times it will run until the algorithm convergence. Another implementation of K-means is Apache Mahout [20] which is used as a machine learning software that allows applications to analyze a large set of data. Mahout uses Apache Hadoop power to solve a complex problem by breaking them up into multi-parallel tasks. Mahout offers three machine learning techniques which are Recommendation, Classification, and Clustering. Recommendations use user's information with community information to determine the likelihood of user's preference. For example, Netflix uses the Mahout Recommendation engine to suggest movies. Classification engine is used for example in classifying spam emails. It uses known data to determine how new data should be classified into a set of existing categories. So every time a user mark an email as 'spam' it directly influences the email Classification engine for providing future email spams. The last Mahout engine is clustering which is used for example to group different news of similar article together. This is mainly used by Google and other search engines. Clustering forms a group of similar data based on common characteristics. Unlike classifications, clustering does not group data into an existing set of known categories. This is particularly useful when the user is not sure how to organize the data in the first place.

\section{Related work}

Analyzing cloud computing performance is an important research topic that leads to several published papers. Map-Reduce clusters are becoming popular for a set of applications [21-23] in which large data processed is stored and shared as files in a distributed file systems.
Emanuel V in [24] presents an analytical model that estimates performance for a Hadoop online prototype using job pipeline parallelism method. In comparison, the projection model proposed in this paper projects performance and runtime using different processor micro-architecture parameters that are important parameters for processor architects to model performance. Furthermore, our model is verified to predict both performance and runtime with $<5 \%$ error margin for all tested cases. The performance projection model we present in this paper is flexible and can be implemented without the need for a simulator and sampling traces.

Dejun et al in [25], propose an approach to evaluate the response time and $\mathrm{I} / \mathrm{O}$ performance. Ibrahim et al in [26], analyze Hadoop execution time on virtual machines. Stewart in [27] compares the performance of several data query languages. All their work is focused on different aspects for analyzing Hadoop performance. Our work complements performance analysis for Hadoop. We also present a prediction analytical model for performance which is the main focus of the research presented in this paper. There are several performance monitoring tools for Hadoop K-means. Salsa [28] for example is a DataNode/TaskTracker log analyzer which provides data and control flow execution on each node. Mochi [29] extracts job execution view from a DataNode/TaskTracker logs.

Therdphapiyanak et al in [30] proposed an implementation using Mahout/Hadoop for a large data set by pre-determining the appropriate numbers of $\mathrm{K}$-means clusters. This is done by describing the appropriate number of cluster and the proper amount of entries in $\log$ files.

Jiang in [31] conducted an in-depth performance analysis for MapdReduce. The research presented optimization methods to improve performance. However, his research does not present an estimation model to translate the optimized methods presented into a performance prediction model.

Esteves et al, in [32] presented detailed performance analysis of Mahout K-means using large data sets running on Amazon EC2 instances. The performance gain in runtime was compared when running on a multi-node cluster.

\section{Performance characterization using Intel Xeon based platform}

Several measurements are conducted to analyze the performance sensitivity for Hadoop K-means iteration using Intel Xeon based platform.

\section{1) Platform Configuration}

The slave node configuration consists of software and hardware configuration. For slave node hardware 


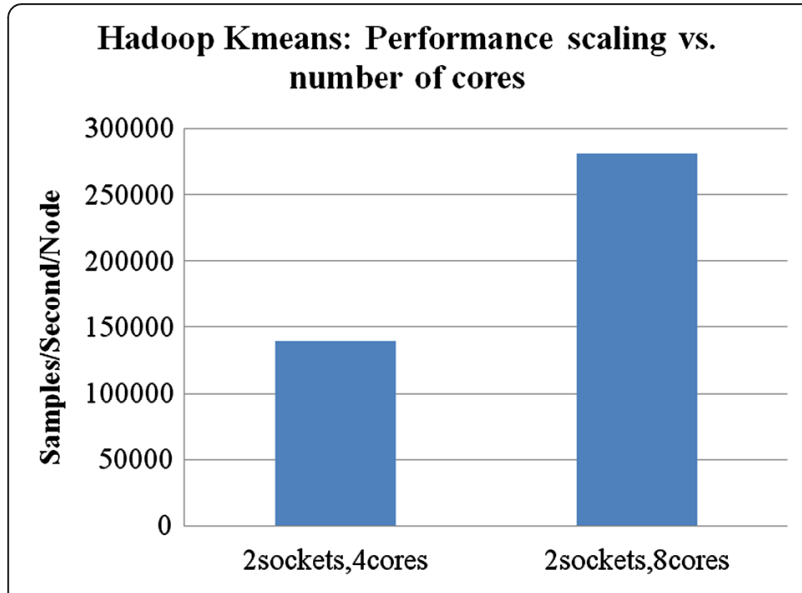

Fig. 1 Performance scaling vs. number of cores Hadoop K-means

configuration, we used 4 Intel platforms with the following setup:

2xIntel Core i7 $\mathrm{CPU}$ at $2.7 \mathrm{GHz}$

32GB Memory (8x 4GB DIMMs) at $1066 \mathrm{MHz}$

Network controller using onboard $1 \mathrm{GbE}$

Seagate disk at 1 TB 7200RPM

HDFS setup using 5x Intel 200GB SSDs on each system

The software configuration for the setup is as follows:

Disabled power management (including C-states)

Disabled Hyper-Threading

Enabled Prefetchers

Operating System used is Red Hat Enterprise Linux

with Apache Hadoop version 1.2.1

1:1 Map slots
1:1 Reduce Slots: 1:1

Heap Size is $2 \mathrm{~GB}$

\section{2) Problem Size requirements}

The workload input size must run and scale on different processor architecture. We have to model it in a way to scale across different processors architectures using the baseline configuration with Intel Core i7 processor. In order to do that, we need the workload input size to be based on the number of active logical threads with fixed memory size per thread. The problem size relation in GB can be stated as

$$
\text { Problem size }=(7 / 8) \times 2 \mathrm{~GB} \times \text { of threads }
$$

The populated memory should scale as close as possible with JVM heap requirements. The metric used for measurement is the number of samples per unit time (seconds) per node. If the problem size varies, the runtime will not be consistent. So the problem size must have a fixed number of sample, for example, a problem size of $28 \mathrm{~GB}$ is equal to $409.6 \mathrm{M}$ sample.

\section{3) Core and Socket Scaling}

For socket scaling, we conduct measurements using one and two processor sockets for the same number of cores. For core scaling, we used two sockets but change the number of cores (4 cores and 8 cores) as shown in Fig. 1.

For core scaling, using four and eight cores, the performance scaling is linear; close to $2 \mathrm{x}$ going from four cores to eight cores. This means that Hadoop K-means performance scales linearly with a number of cores.

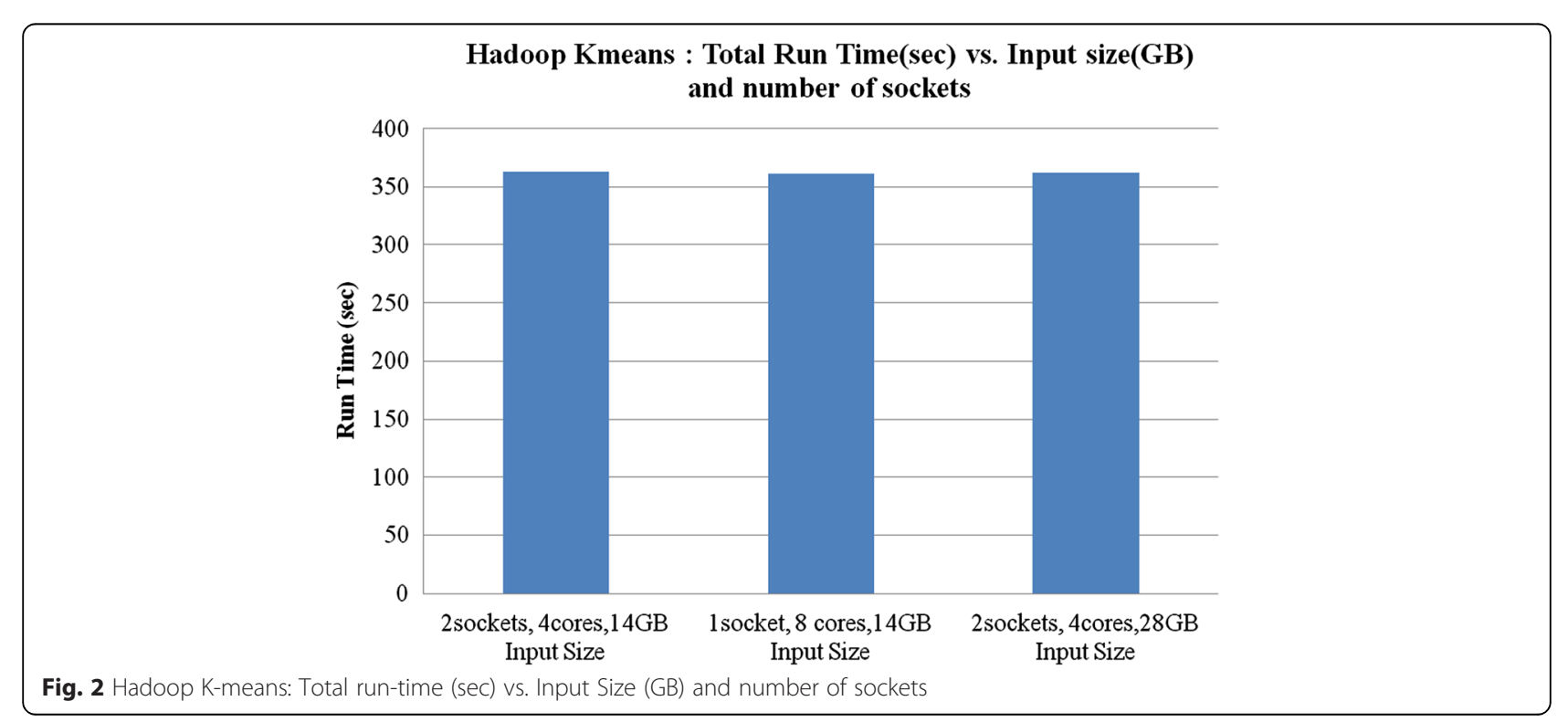




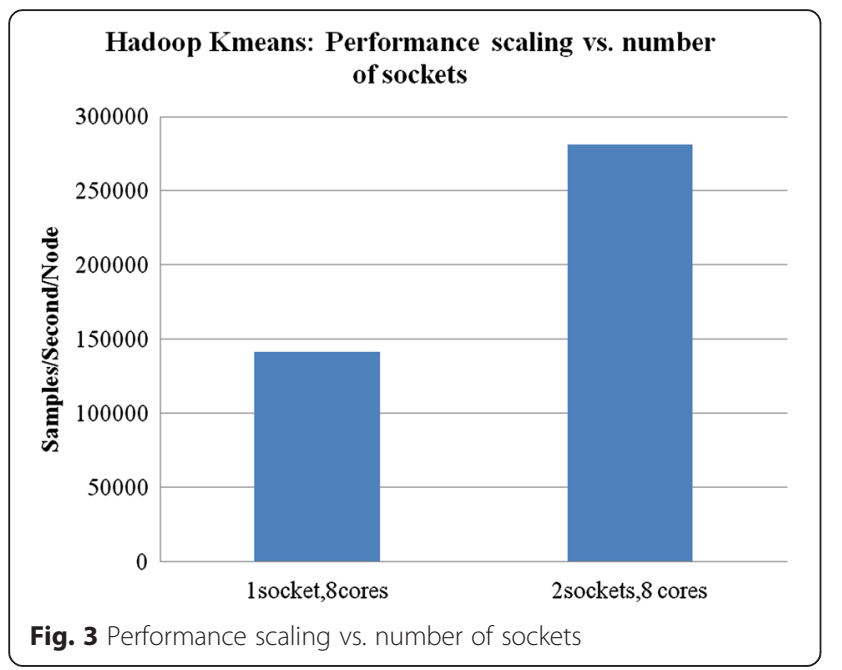

For socket scaling, using one and two sockets with eight cores on both, the performance scaling (Samples/Second/Node) shows a linear scaling is about 1.99x. We conclude that both socket and core scaling is $2 x$. The core scaling was adjusted accordingly using "Active Core Count" in BIOS while the socket scaling is achieved using the physical removal of a processor with associated memory. The Cycles-Per-Instruction (CPI) overall for the benchmark using different input sizes (14 GB and $28 \mathrm{~GB}$ ) calculated is about 0.52 for 1 socket $/ 8$ cores, 2 sockets $/ 8$ cores, and 2 sockets $/ 4$ cores. The Execution Length (EL) for different configurations is constant $\sim 262,000$. The EL is defined by the total number of instructions executed per different cores. In this case, it is constant and doesn't change with respect to the change in the number of cores. The total runtime for different input sizes and different configurations is constant about 362 seconds as shown in Fig. 2.

This shows that the total runtime (seconds) is almost constant relative to a different number of sockets, the number of cores and the input data size. What change is the performance metric defined as Samples/ Second/Node for different configurations as previously shown in Fig. 1 and Fig. 3. Next, we analyze core and socket scaling with respect to memory bandwidth $(\mathrm{GB} / \mathrm{sec})$ and performance as shown in Fig. 4.

From Fig. 4 we conclude that performance is correlated with memory bandwidth. That is the scaling in performance between 1 socket/ 8 cores/14GB input size to 2 sockets/4 cores/28GB is about $2 x$. Same is true for memory bandwidth; the scaling for the same configurations is also about $2 \mathrm{x}$.

\section{4) Core Frequency Scaling}

Core frequency scaling is required so we can analyze how performance behaves with respect to higher core frequencies as shown in Fig. 5. The scaling between two or three core frequencies will indicate the performance change between these measured data points; so that we can use to model the performance with respect to the frequency change.

From Fig. 5, the performance is measured as a number of samples per second per node (samples/second/mode). The performance scaling from $2.1 \mathrm{GHz}$ to $2.4 \mathrm{GHz}(2.4 /$ $2.1=1.14)$ is about $1.13 \mathrm{x}(249100 / 220000)$ in performance, and from $2.4 \mathrm{GHz}$ to $2.7 \mathrm{GHz}(2.7 / 2.4=1.125)$ the performance scaling is about $1.12 \mathrm{x}(281300 / 249100)$.

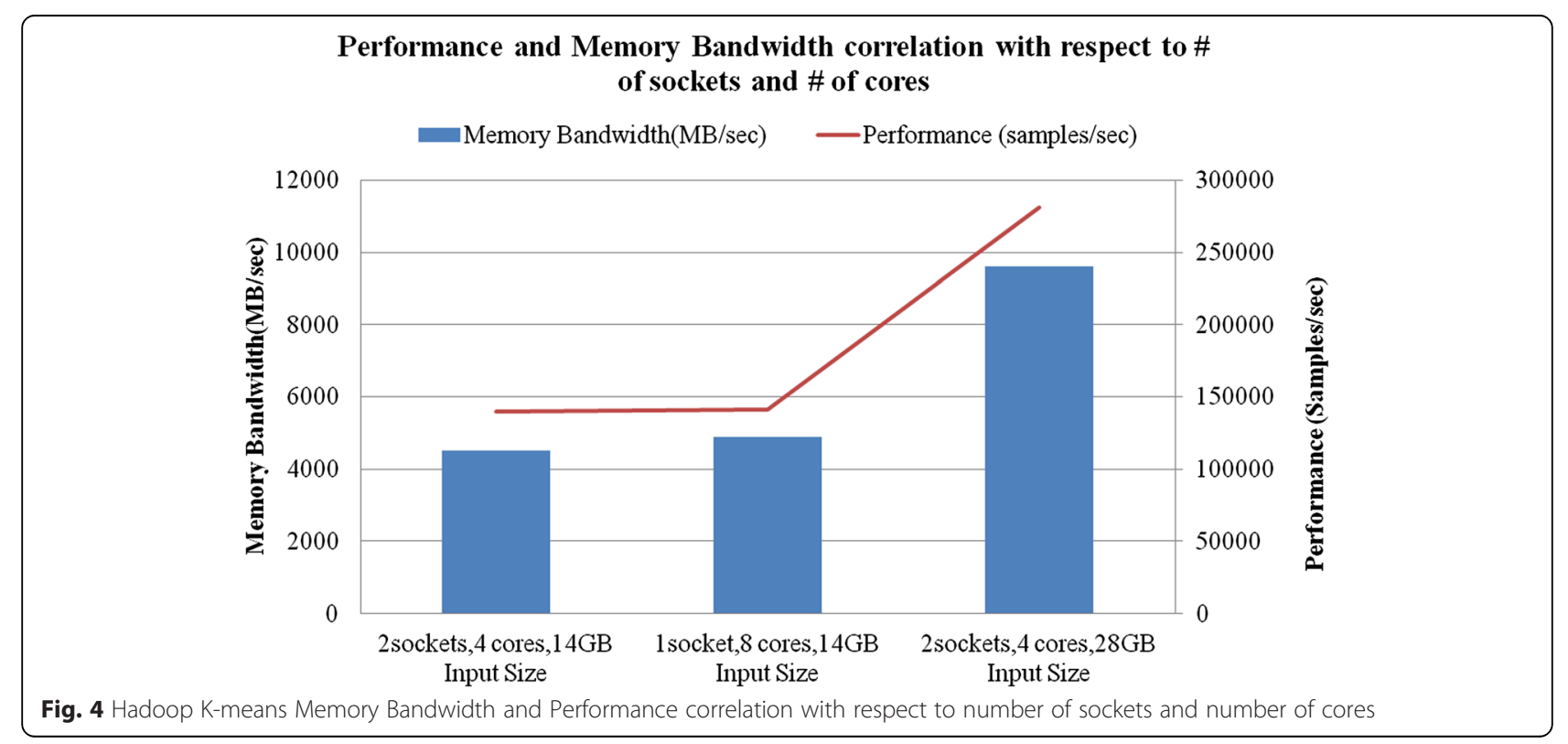


Hadoop Kmeans: Performance scaling relative to different core frequencies

Fig. 5 Hadoop K-means: Performance vs. Core Frequency Scaling

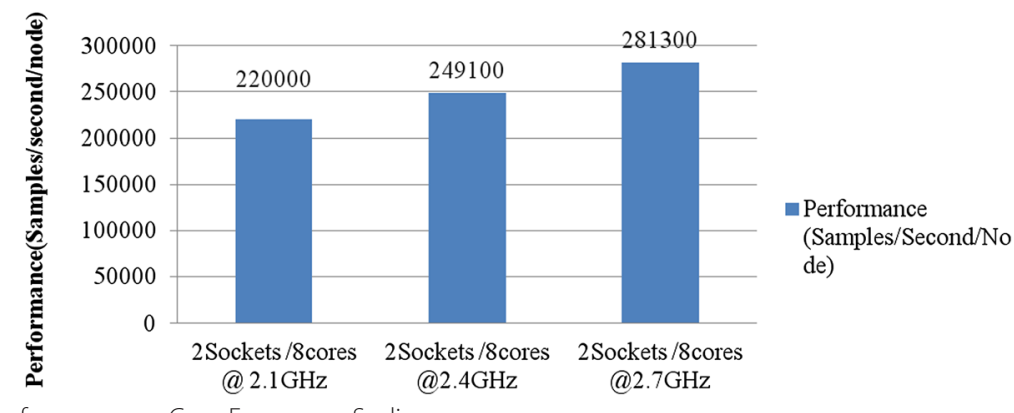

However, the performance scaling from $2.1 \mathrm{GHz}$ to $2.7 \mathrm{GHz}(1.28)$ is about $1.27 \mathrm{x}(281300 / 220000)$. This shows that there is an excellent scaling between core frequency and performance so we can use this scaling factor for core frequency in the performance model. Memory bandwidth scales almost linearly with core frequency as shown in Fig. 6.

The scaling rate from $2.1 \mathrm{GHz}$ to $2.4 \mathrm{GHz}$ is $1.14 \mathrm{x}$ while the scaling rate for memory bandwidth is $8670 / 7600=$ $1.14 \mathrm{x}$. Similarly, the scaling for $2.1 \mathrm{GHz}$ to $2.7 \mathrm{GHz}$ is $1.28 \mathrm{x}$ and the memory bandwidth scaling is $9600 / 7600=1.26 x$. This shows that the scaling factor for memory bandwidth with respect to the scaling factor for core frequency is linear.

5) Hyper-Threading/Simultaneous Multi-Threading Scaling
Enabling the processor Hyper-Threading (HT) feature will enable an active core to execute two threads per core instead of one thread or Single Thread (ST). In our performance characterization, we found that enabling Simultaneous Multi-Threading (SMT) and scaling the workload with respect to thread count shows an average of $20 \%$ increase in performance with all cores active.

In Fig. 7, SMT scaling for 2 sockets/4 cores shows $1.2 \mathrm{x}$ improvements in performance from Single Thread (ST) to $\mathrm{HT}$ with $1 \mathrm{~GB}$ heap size. It also shows a $1.24 \mathrm{x}$ performance improvement from ST to HT with 2GB Heap size. For 2 sockets/ 8 cores, the improvement from ST to HT with $1 \mathrm{~GB}$ heap size is $1.17 \mathrm{x}$ and for HT with $2 \mathrm{~GB}$ heap sizes its $1.22 \mathrm{x}$ in performance improvement. Given that HT is enabled, the CPI for the kernel increases with an average of $40 \%$ as compared to HT off, but the overall CPI is 0.97 on average.

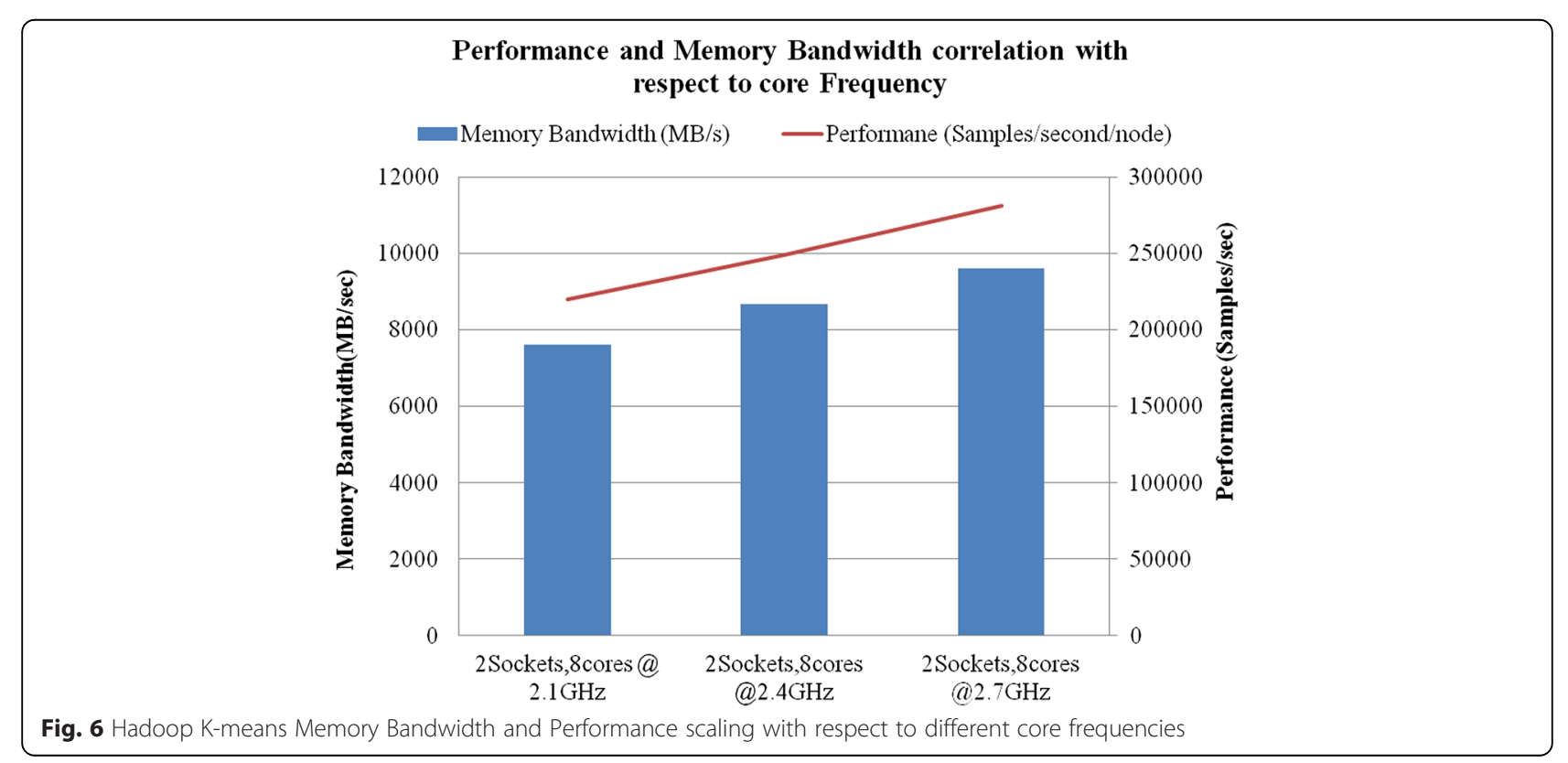




\section{Performance scaling vs. Simultaneous Multi Threading Scaling with different heap sizes}

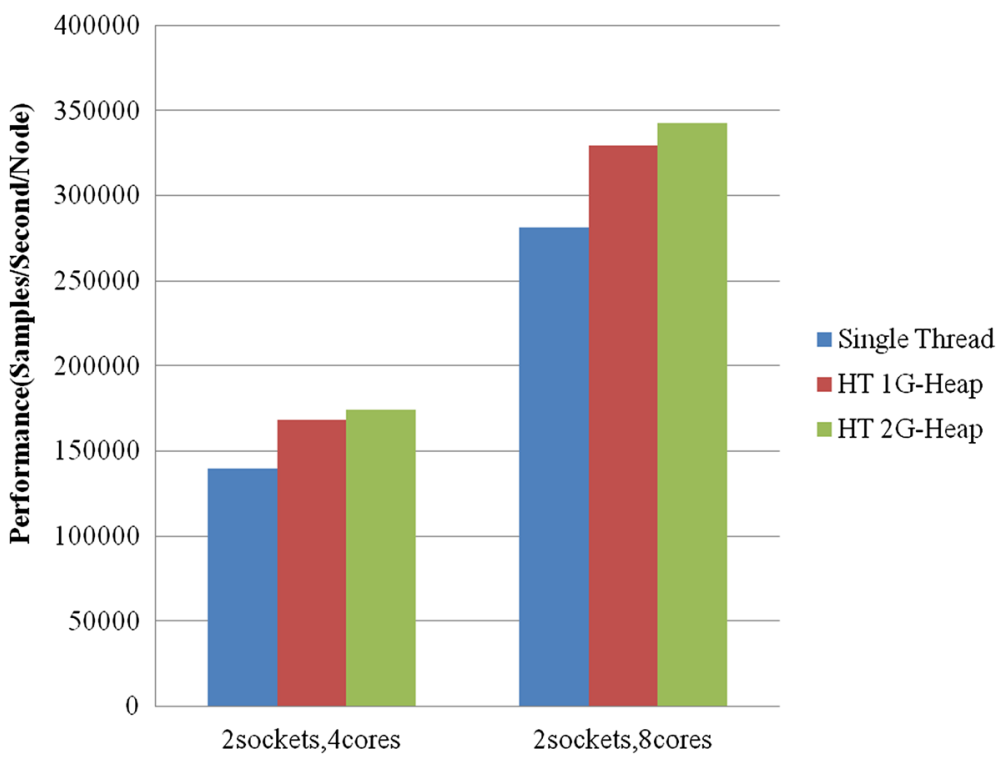

Fig. 7 Hadoop K-means: Performance vs. Simultaneous Multi-Threading scaling with different heap sizes

6) Last Level Cache Scaling

The capacity of Last Level Cache (LLC) does not have any impact on performance improvement. If the cache size is increased from $10 \mathrm{MB}$ to $20 \mathrm{MB}$, the performance improvement is only $1.01 \mathrm{x}$ as shown in Fig. 8 using 2 sockets $/ 4$ and 8 cores processor configuration. So for Hadoop K-means workload it is not bounded by LLC. We conclude there is no performance improvement even if LLC size is increased above $20 \mathrm{MB}$.

The Message Passing Interface (MPI) is directly related to the LLC size. For $10 \mathrm{MB}$ LLC size, the overall LLC MPI measured is 0.00111 using 2 sockets with 4 cores. For $20 \mathrm{MB}$ LLC size, the MPI measured is 0.00097 , this shows that MPI is affected by LLC size with about $14 \%$ decrease with LLC $20 \mathrm{MB}$ versus LLC $10 \mathrm{MB}$ which is expected. For memory bandwidth, there is about $7 \%$ lower in memory bandwidth utilization for the same performance with larger LLC.

7) Data Input Size Scaling

Data input size is a key factor that impacts performance for Hadoop K-means. We scaled input size and analyzed the change in execution run-time. The result is

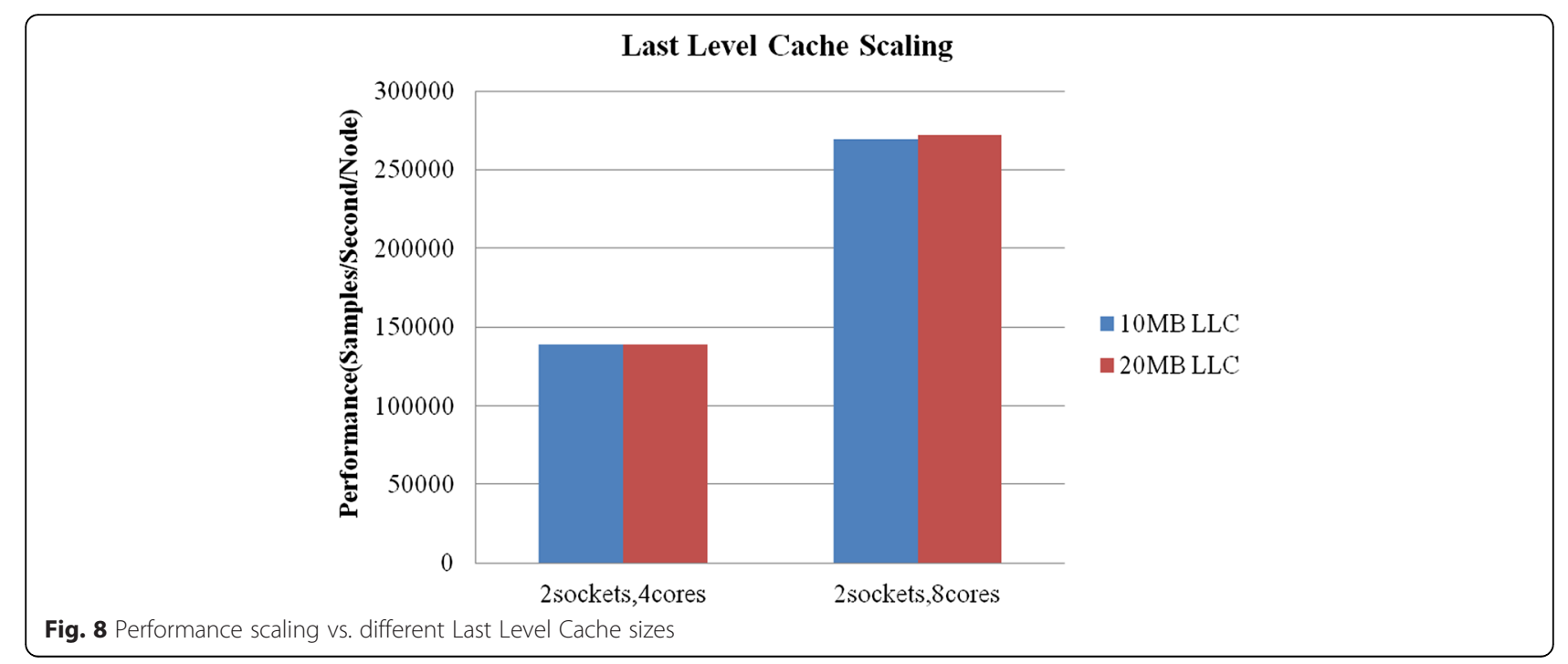




\section{Run Time (sec) versus Input size (GB) for different Memory Sizes}

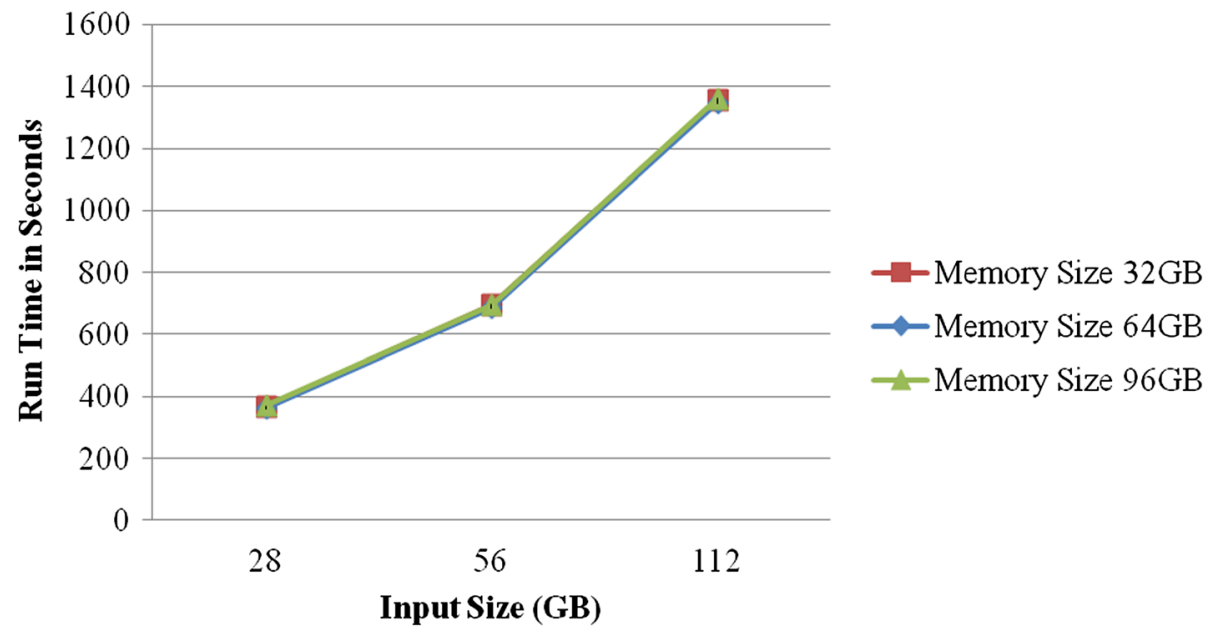

Fig. 9 Total run-time (sec) vs. different input sizes (GB) for different memory sizes (GB)

an increase of twice the input size results in about $1.9 \mathrm{x}$ increase in run-time. For example, the scaling factor for $56 \mathrm{~GB} / 28 \mathrm{~GB}$ is $2 \mathrm{x}$, and the scaling factor for run-time (695 sec/367 sec) is 1.9x. Figure 9 shows the input size scaling with respect to total run-time for different memory sizes. In this case, the memory size does not have any impact on total run-time, with less than $1 \%$ variation for memory size 32GB, 64GB, and 96GB.

In summary, a $2 \mathrm{x}$ increase in input size will result in about $1.9 \mathrm{x}$ increase in execution time regardless of memory size. Figure 9 shows even more linear scaling going from 56GB to $112 \mathrm{~GB}$ input size.

8) Memory and Heap Size Scaling

For memory scaling, there is almost a $1 \%$ run-time variation between different memory sizes. This indicates there is no performance and run-time variation for Hadoop K-means for these three memory sizes (32GB, 64GB, and 96GB) as shown in Fig. 10.

\section{Hadoop Kmeans: Total run time(sec) vs. different Memory and input size(GB)}

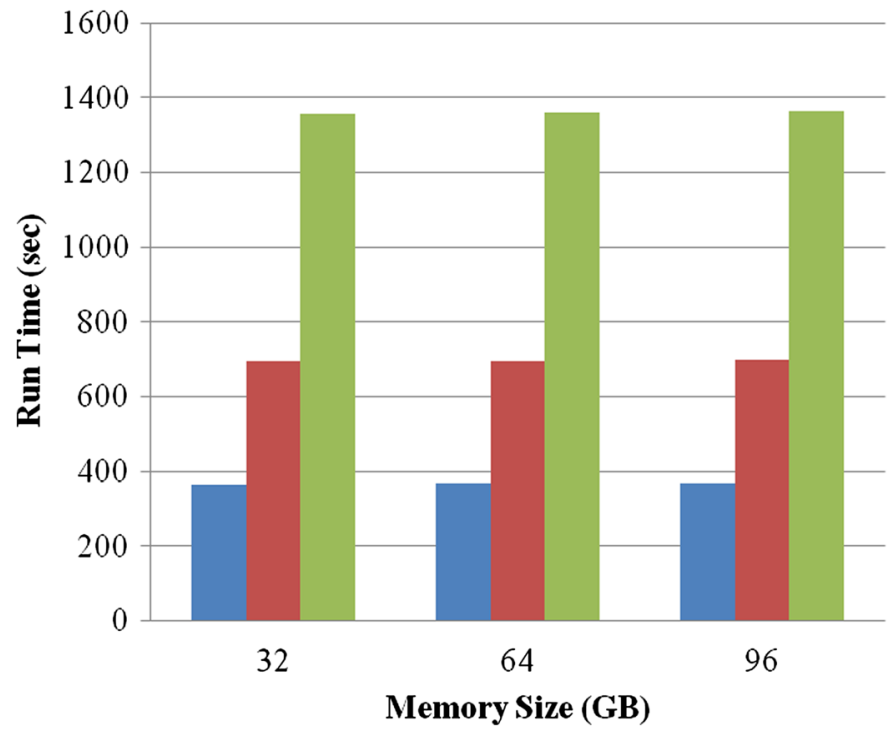

Input Size $28 \mathrm{~GB}$

Input Size $56 \mathrm{~GB}$

Input Size 112 GB

Fig. 10 Total run-time (sec) vs. different Memory and input sizes (GB) 
The run-to-run variation for the run-time data collected for memory scaling is in the range of $1 \%-3 \%$ which is an expected run-to-run variation range. For heap size scaling, we used heap size of $1 \mathrm{~GB}, 2 \mathrm{~GB}$, and 4GB. The run-time is almost the same for different input size as shown in Fig. 11.

\section{Performance characterization using AMD interlagos platform}

In this section, we implement performance analysis characterization for Hadoop K-means iteration using AMD Interlagos platform.

\section{1) AMD Platform Setup}

The slave node configuration consists of software and hardware configuration. For slave node hardware configuration, the following setup is used:

4x AMD Interlagos platforms (Bulldozer core)

Two different chassis: $2 \mathrm{x}$ HP Proliant and $2 \mathrm{x}$

Supermicro

CPU: 2x 2.60GHz ITL

Memory: Fixed at $1066 \mathrm{MHz}$ for all configurations

$32 \mathrm{~GB}=4 \mathrm{x} 8 \mathrm{~GB}$ DIMMs (1-socket) and 64GB $=8 \mathrm{x} 8 \mathrm{~GB}$

DIMMs (2-socket)

NIC: Onboard 1GbE (only 1 port in use)

For disk configuration, the System disk used is Seagate 1 TB 7200RPM (holds no HDFS data). The HDFS: 4x Intel 200GB SSDs on each system. All disks attached via an SAS controller.

For slave node software configuration, the following setup is used:
Power Now: Enabled, but frequency fixed via On Demand governor and Core Performance Boost disabled

Prefetchers: Enabled

Operating System: Red Hat Enterprise Linux 6.1 with

Kernel version 2.6.32

Java: Sun 1.6.0_25

Hadoop distribution: 1.0.2 snapshot (based on Apache distribution)

Map Slots: 1:1 with active logical threads

Reduce Slots: 1:1 with active logical threads

Heap Size: $2 \mathrm{~GB}$

2) Problem Size Requirements

The workload needs to scale across all testing domains using different processor architectures. So the approach is to use a workload input size based on a number of active logical threads with fixed memory size per thread as indicated in Eq (1). The populated memory also scales as close as possible with JVM heap requirements. The metric used for measurement is samples/sec/node. The run-time is not a consistent metric if we are varying the problem size. A given problem size has a fixed number of samples (unit of work). Ex. 28GB problem size per node (without replication $)=409.6 \mathrm{M}$ total samples. The metric calculated is Total Samples/Run-time/\# of Nodes.

3) Hadoop K-means Performance Scaling with respect to thread Count

This experiment is implemented to scale performance with respect to thread count. The measurements were

\section{Total run time(sec) vs. Heap Size (GB) and different input size(GB)}

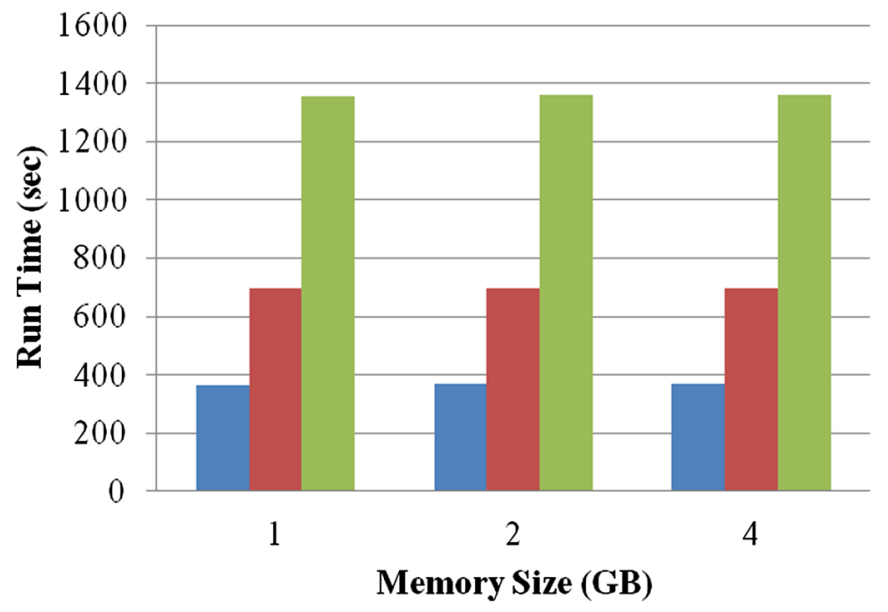

Input Size $28 \mathrm{~GB}$

Input Size $56 \mathrm{~GB}$

Input Size $112 \mathrm{~GB}$

Fig. 11 Total run-time (sec) vs. heap and input size scaling 


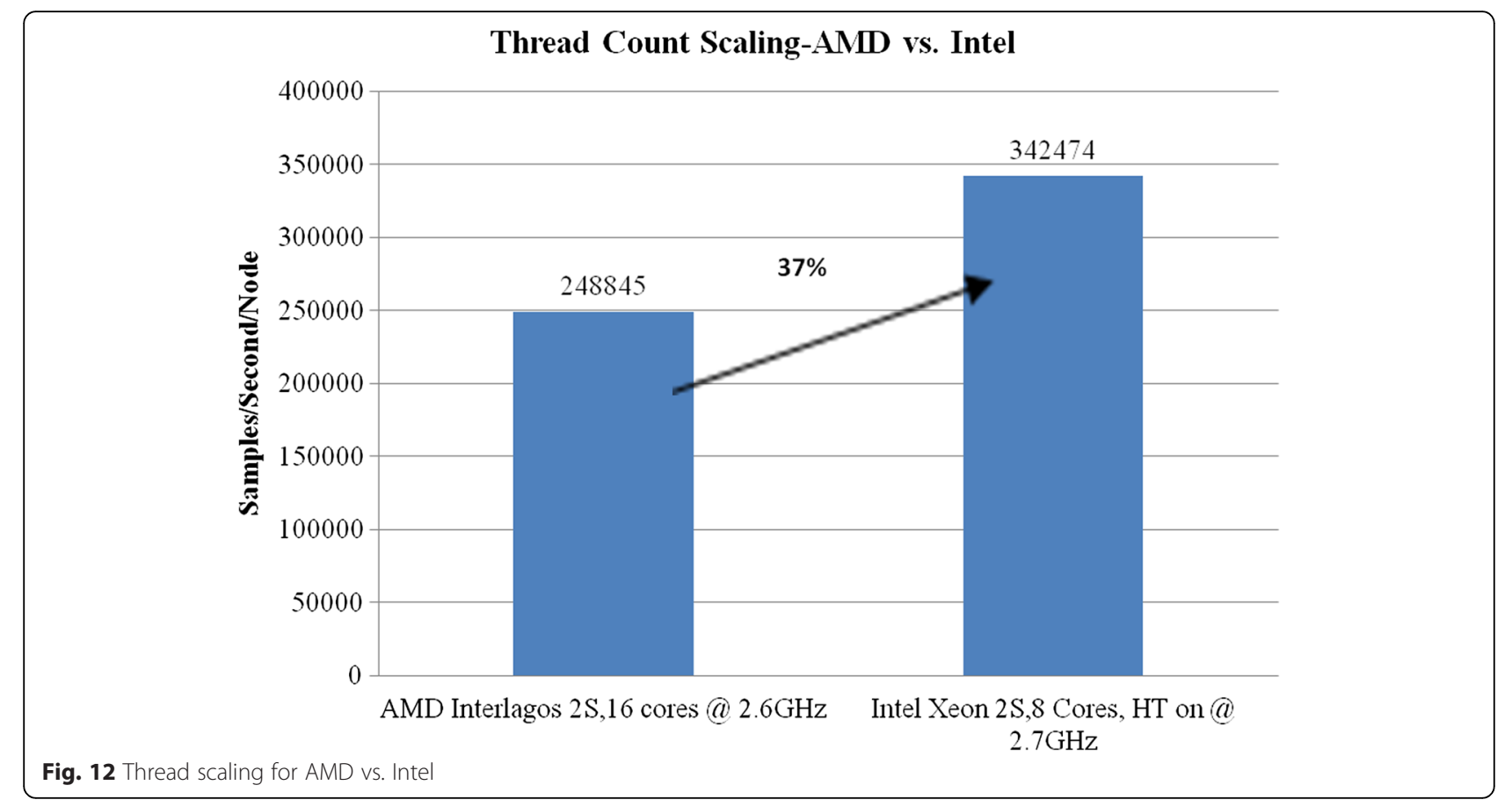

taken using Intel Xeon processor (2 sockets, 8 cores, Hyper-Threading $\mathrm{On}$, at $2.7 \mathrm{GHz}$ core frequency) which gives a total of 32 threads versus AMD Interlagos (2 sockets, 16 cores, at $2.6 \mathrm{GHz}$ core frequency) which also gives a total of 32 threads. Comparing the performance for these two configurations, we conclude that there is a $~ 37 \%$ lead for Intel Xeon compared to AMD Interlagos using the same thread count as shown in Fig. 12. Given the slight difference in core frequency $(2.6 \mathrm{GHz}$ vs. 2.7GHz), our Hadoop K-means frequency scaling assessment indicates that the performance will be close to $34 \%$ instead of $37 \%$.

The total run-time for Intel vs. AMD shoes that Intel Xeon total run-time is $35 \%$ less (smaller the better) compared to AMD run-time as shown in Fig. 13. This is implemented for the same number of threads (32).

The CPI for Intel Xeon is measured at 0.96 versus 1.26 for AMD which means Intel CPI is $\sim 24 \%$ lower as compared to AMD.

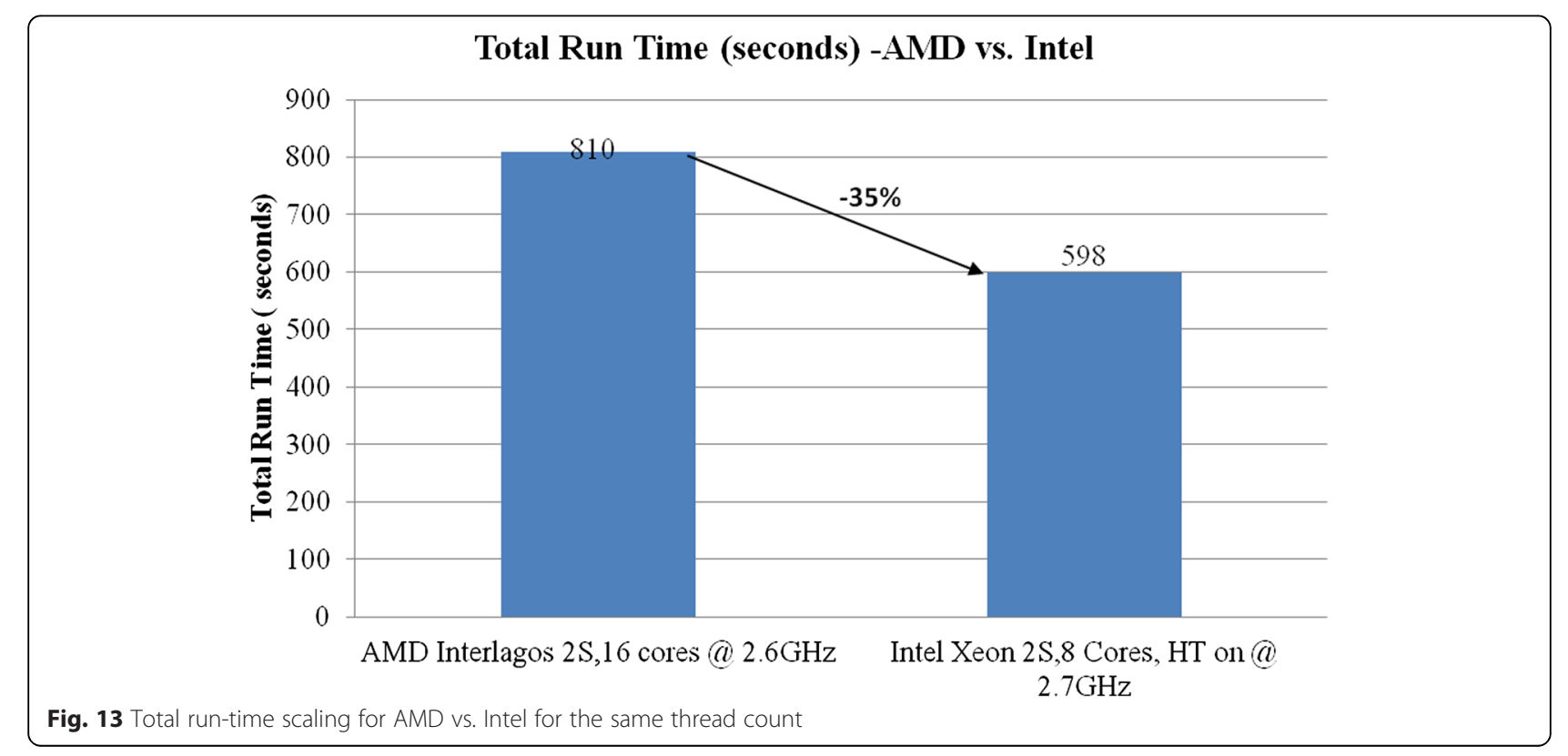




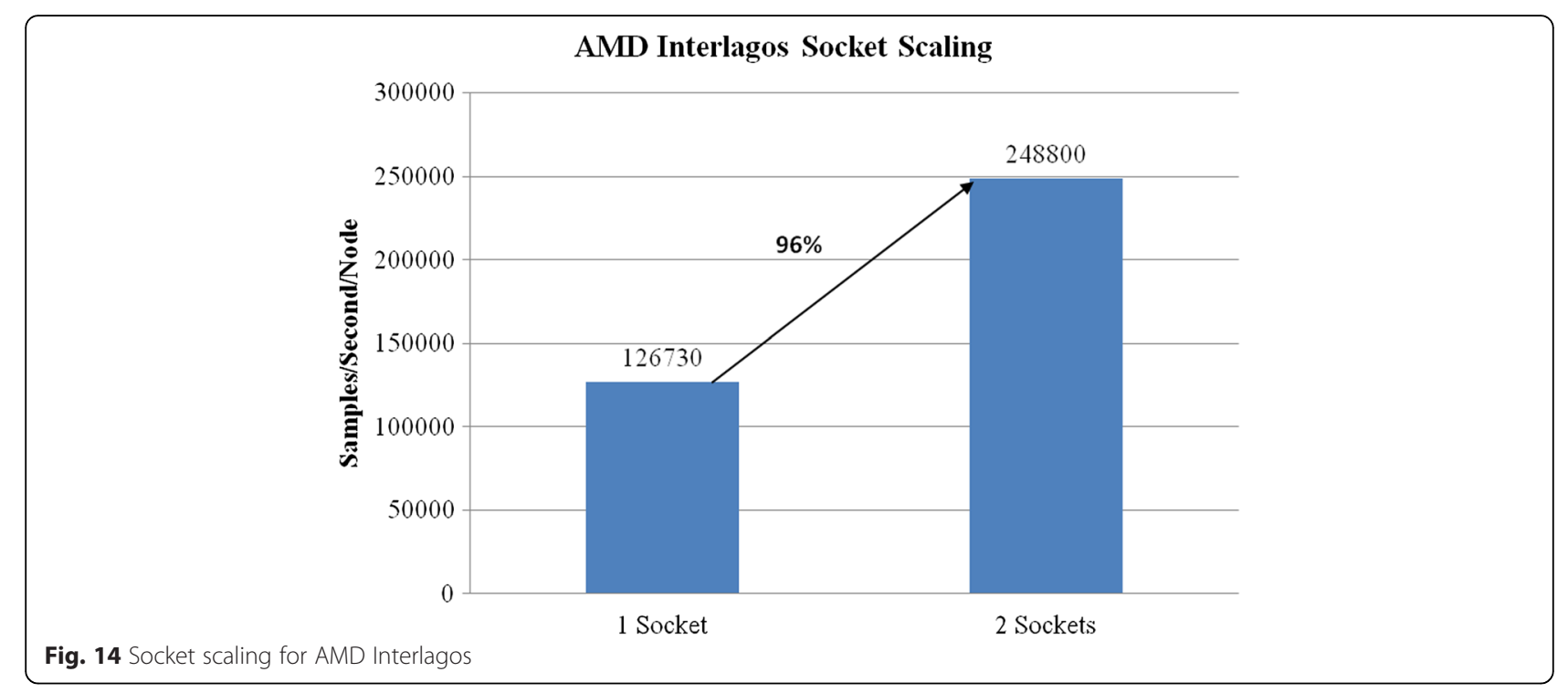

4) Hadoop K-means Performance scaling with respect to thread count

Performance change with respect to a change in the number of sockets was implemented on AMD Interlagos system for one and two sockets.

From Fig. 14, we conclude that there is a linear scaling between one and two sockets on AMD Interlagos system. The performance rate change is $96 \%$ going from one to two sockets. For Intel Xeon system, the performance is also about $98 \%$ between one and two sockets configuration. This confirms the Hadoop K-means scales linearly with respect to the number of processor sockets. For the CPI scaling as the number of sockets doubles, the CPI change from one to two sockets is relatively flat around 1.22 for one socket versus 1.26 for two sockets. We conclude that CPI does not change significantly as the number of sockets doubles.

5) Hadoop K-means Performance Scaling for AMD with respect to core count using 2 sockets

Hadoop K-means performance scaling for AMD Interlagos using 2 sockets processor shows a linear scaling with respect to a change in a number of cores as shown in Fig. 15. The performance almost doubles when the number of cores doubles (Fig. 16).

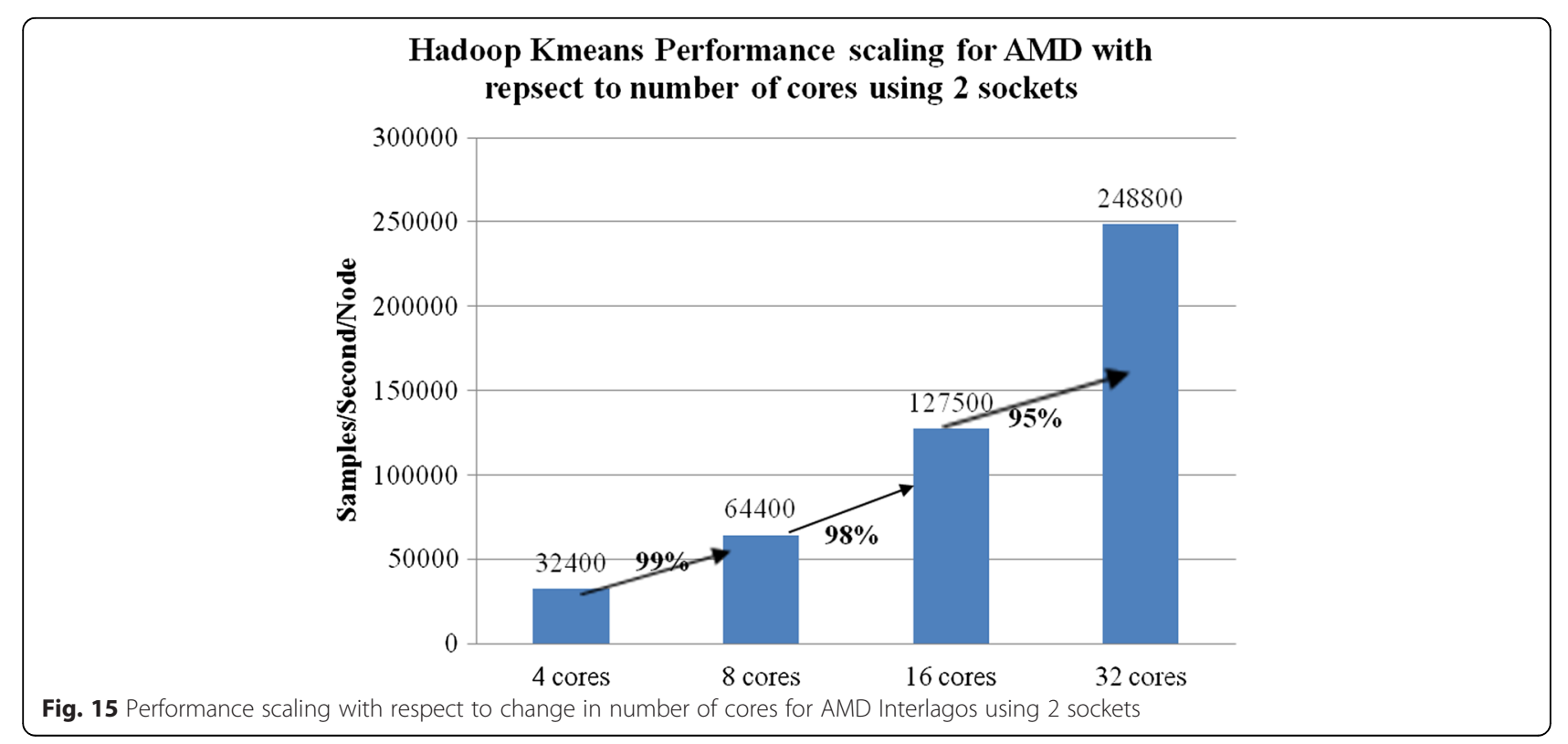




\section{Hadoop Kmeans CPI scaling with repsect to number of cores}

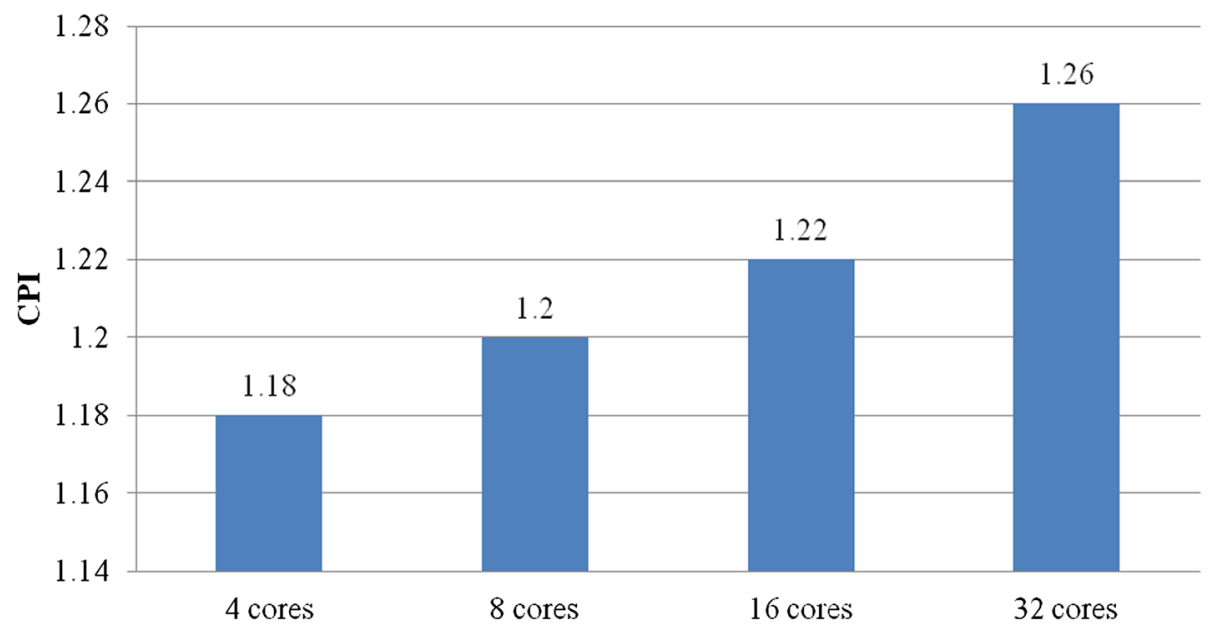

Fig. $16 \mathrm{CPI}$ scaling with respect to change in number of cores for AMD Interlagos

The same measurement was implemented on Intel Xeon processor, and a similar conclusion can be concluded for Intel Xeon as performance doubles when the number of cores doubles. There is a slight increase in CPI when the number of cores doubles.

We conclude there is a slight increase in CPI when the number of cores increases at a non-linear rate.

6) Hadoop K-means Performance Scaling for AMD with respect to core frequency

The performance for Hadoop K-means scales almost linearly with respect to change in core frequency as shown in Fig. 17. For example changing core frequency from $2.0 \mathrm{GHz}$ to $2.3 \mathrm{GHz}(2.3 / 2.0=1.15$ or $15 \%)$ results in a $12 \%$ increase in performance $(225000 / 200100=$ 1.12). Similarly changing the core frequency from $2.3 \mathrm{GHz}$ to $2.6 \mathrm{GHz}(2.6 / 2.3=1.13$ or $13 \%)$ will result in 1.105 change rate in performance $(248800 / 225000=$ 1.105 or $10.5 \%)$. Note that the frequency scaling was achieved through Linux governor.

7) AMD Interlagos core and cluster scaling using one socket

The objective for analyzing performance with respect to a number of clusters and a number of cores is to understand the core-per-cluster impact on performance. From

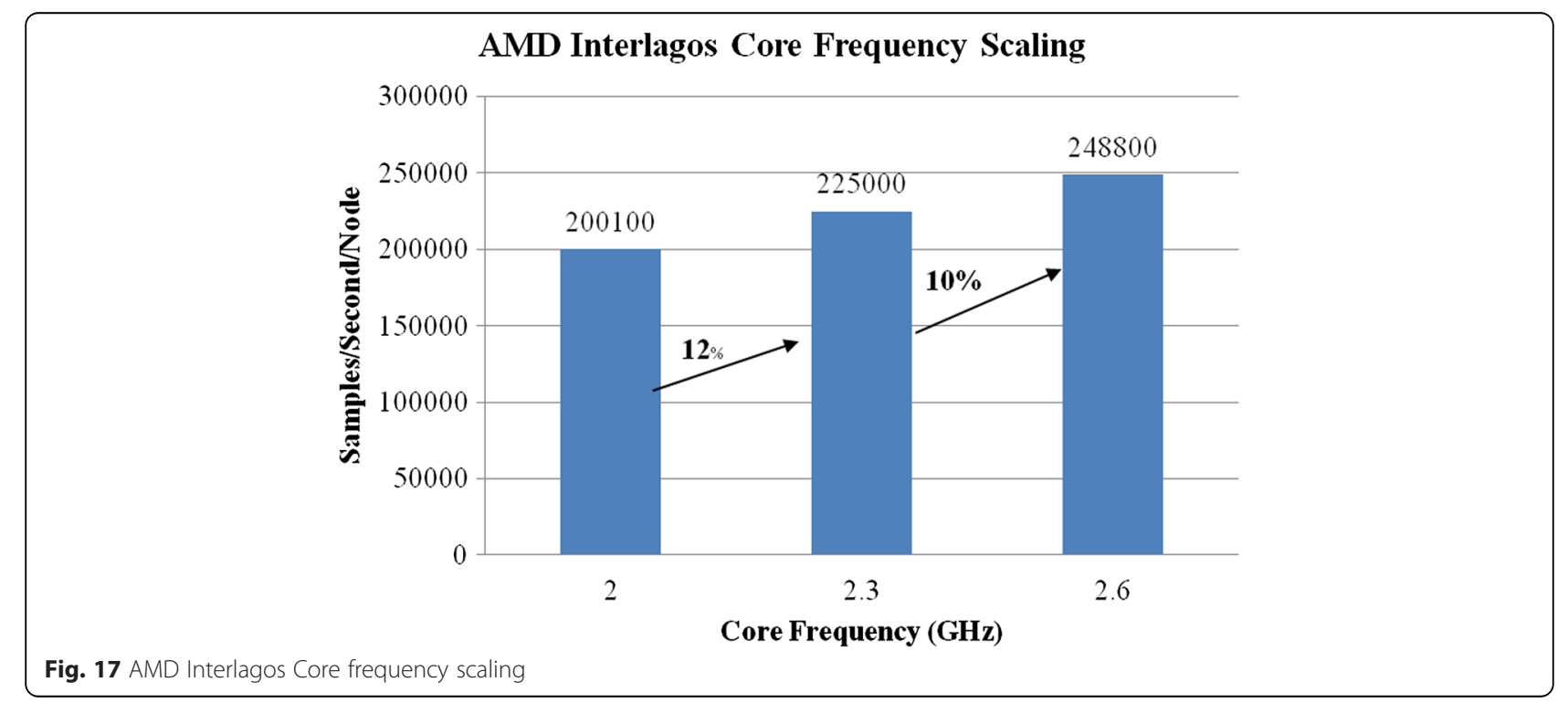


Fig. 18, we conclude there is a performance benefit for additional core per cluster. For example, in four cluster configuration, the change from one thread to two threads results in about $60 \%$ increase in performance. Similarly for eight clusters, the performance benefits going from one thread to two threads is also about $60 \%$. However in one cluster configuration, the performance benefit going from one to two threads is almost doubled ( $>100 \%)$. We can conclude that there is a performance benefit for additional core-per-cluster for most configurations.

There is an increase in performance going from one cluster to four clusters in a range of $4.4 \mathrm{x}$, and the performance increases by $\sim 2.2 \mathrm{x}$ going from four clusters to 8 clusters as shown in Fig. 18.

\section{Performance model}

In this section, we discuss a detailed processor performance projection model for Hadoop K-means based on the performance characterization we did in Section IV is used to derive the performance model. We start with defining the general equation for performance, the performance (samples/second) relation is derived as

$$
\text { Performance }=\frac{\text { core frequency } \times \text { Total } \# \text { of cores }}{\text { Execution Length } \times \text { CPI }}
$$

and the total run-time in seconds is given by

$$
\text { Run Time }=\frac{\text { Input Size }}{\# \text { of nodes } \times \text { Performance }} .
$$

The Execution Length (EL) is defined by the total number of instructions executed divided by measured performance baseline given as

$$
\text { Execution Length }=\frac{\text { of instructions executed }}{\text { measured performance }} .
$$

The Cycles-per-Instruction is given as

$$
\begin{aligned}
C P I= & \text { CoreCPI }+0.5 \times M P I \\
& \times \text { Memory Latency, }
\end{aligned}
$$

where the core CPI is defined as the CPI without the memory stall cycles added. In other words, it is the CPI with no cache misses in the Last Level Cache. Given that this scenario is not realistic, we have to add the cycles generated from memory misses which is the MPI multiplied by memory latency. The '0.5' factor used in Eq (5) to multiply the memory latency and MPI, is caused by memory cache miss which is referred to as a memory blocking factor. We expect the memory blocking factor to range from 0 to 1 for most processors. In case it is equal to 0 , this means there are no memory misses at all which means that the CPI is equal to the CCPI. This is not a realistic scenario for this workload. On the other hand, if the memory blocking factor is ' 1 ', it means that there is $100 \%$ miss rate, which is not a realistic scenario. Based on statistical analysis for memory a 0.5 value for blocking factor is used for Hadoop K-means. The model is verified to project performance (samples/second) and

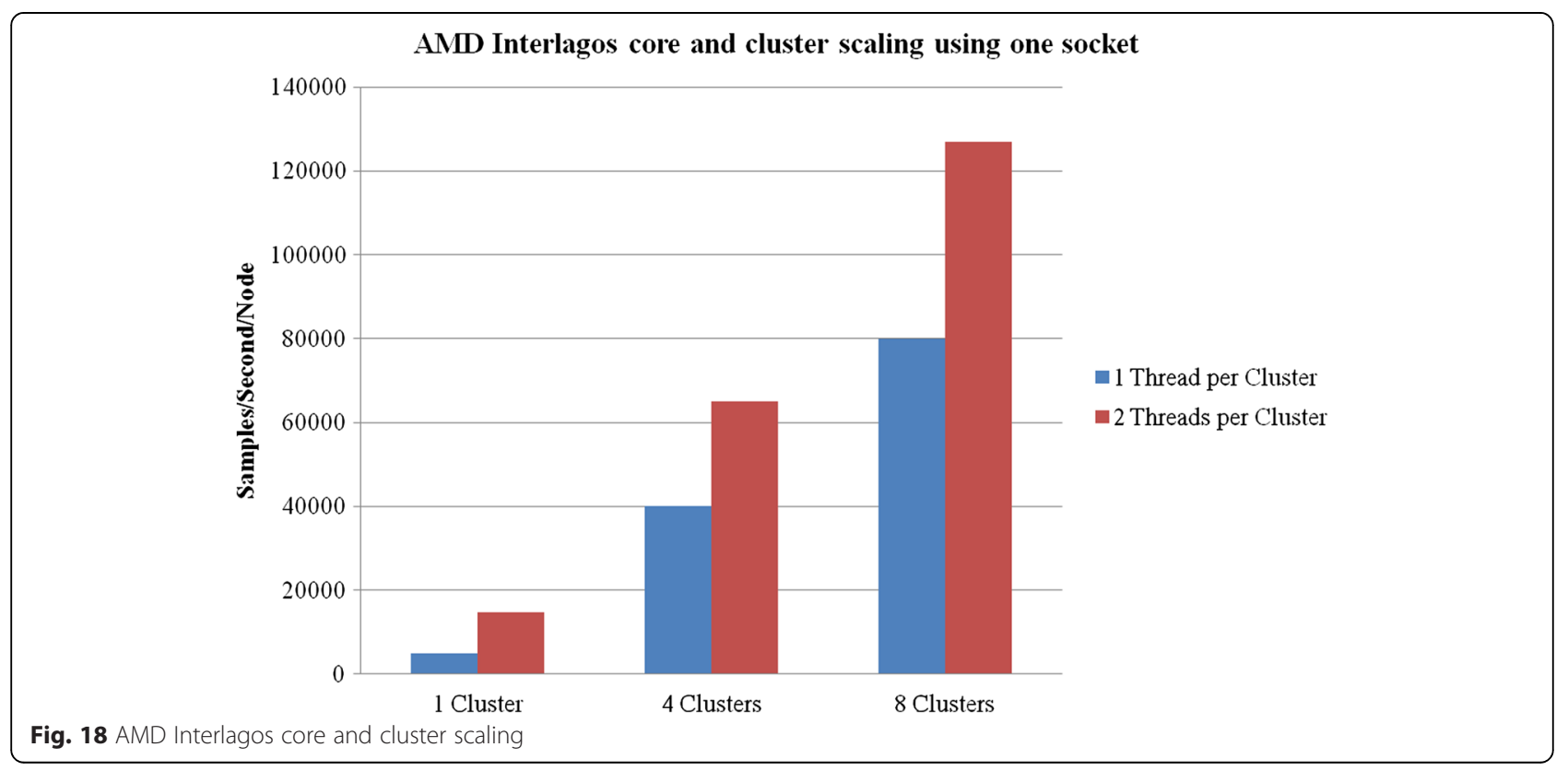




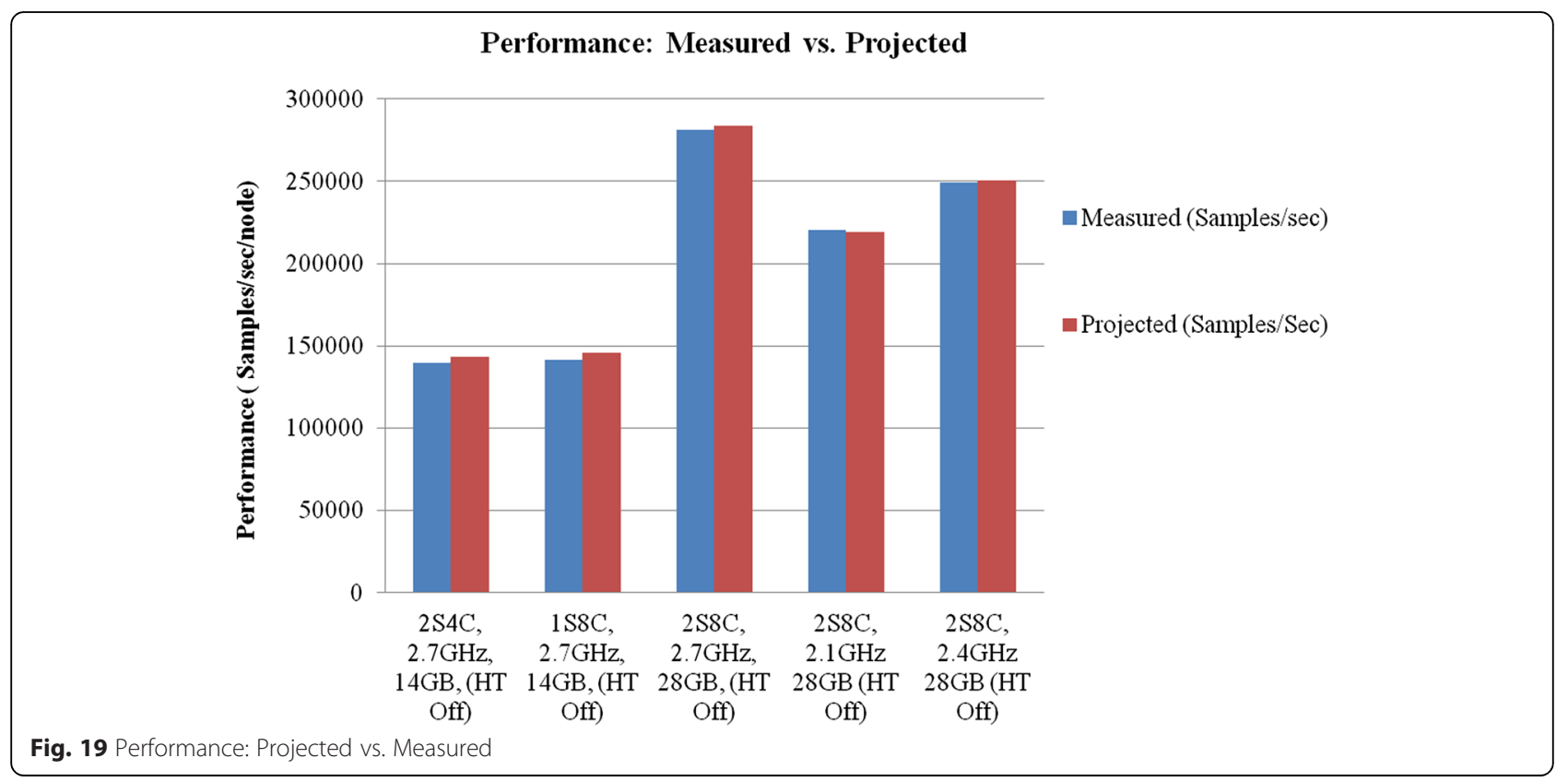

run-time(seconds) with $<5 \%$ error margin for all tested cases as shown in Fig. 19 and Fig. 20.

From Fig. 19, the performance measured is compared to performance projected by the model. We verified different processor configurations such as different number of sockets, different number of cores, different core frequency, and different input sizes, with Hyper-threading set to off. All these variables are included in the performance model. The error variation is within expected range of $<5 \%$. Among all tested configurations in Fig. 19, the peak performance achieved is for 2 sockets, 8 cores, $2.7 \mathrm{GHz}$ core frequency with $28 \mathrm{~GB}$ input size.

For modeling run-time, the highest run-time is expected for the configuration with lowest core frequency which in this case is $2.1 \mathrm{GHz}$ as shown in Fig. 20. All tested cases for run-time (measured vs. projected) shows an error margin of $<5 \%$.

\section{Conclusion}

In this paper, we presented a detailed performance characterization analysis for Hadoop K-means using Intel and AMD based processors. We also proposed a projection model for Hadoop K-means workload. The projection model is verified to project performance and runtime with $5 \%$ error margin for all tested cases. The model is flexible to accept any changes in processor micro-architecture parameters and estimate performance or runtime. The model does not require any simulation which in turn requires trace based sampling for the workload. In future work, we can implement the same approach for different Hadoop framework workloads such as word count and

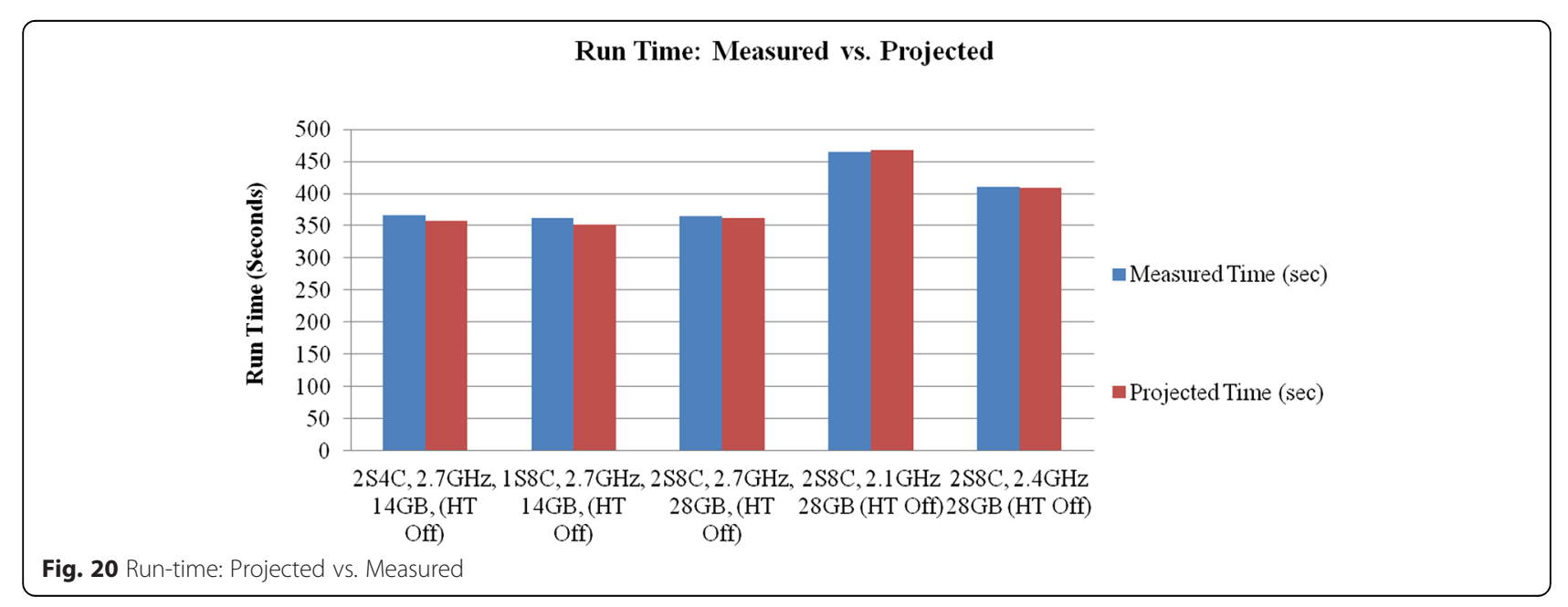


implement a full details performance characterization. The model can be expanded to include IO latency such as disk and network latency. The focus of this paper is on the processor performance excluding any IO latency, this is why the input size selected was 28GB which is less than the system memory size of 32GB. For AMD Interlagos versus Intel Xeon performance analysis, we conclude that there is about $38 \%$ better performance for Intel Xeon as compared to AMD Interlagos. The socket and core scaling is almost linear in most measured cases, the sample conclusion applied to Intel Xeon Processor. For cluster-percore scaling, there is about $60 \%$ increase in performance for AMD Interlagos processor.

\section{Competing interests}

The authors declare that they have no competing interests.

\section{Authors' contributions}

The research presented in this paper is the result of individual work. The author read and approved the final manuscript.

\begin{abstract}
Authors' information
Joseph A. Issa Dr. Issa joined the faculty of engineering at Notre Dame University in October 2013 as an assistant professor in the department of Electrical, Computer and Communication Engineering (ECCE). Dr. Issa research areas of interest include processor architecture, performance and power modeling for different processor architectures using different cloud computing and high-performance computation workloads. He teaches a number of subjects at Notre Dame University including computer processor architecture (MIPS, x86, and ARM), microprocessors, PIC microcontrollers and digital logic design. He received his B.E in computer engineering from Georgia Institute of Technology in 1996. He obtained his master's degree in computer engineering at San Jose State University in California in 2000 and received his Ph.D. in Computer Engineering from Santa Clara University in California in 2012.
\end{abstract}

Received: 10 August 2015 Accepted: 6 March 2016

Published online: 17 March 2016

\section{References}

1. Mak WW and Lundstrom SF (1990) Predicting performance of parallel computations. IEEE Trans. Parallel Distributed Systems, Online Journal

2. Mell P, Grance T (2011) The NIST definition of cloud computing. NIST Special Publication, online (800-145)

3. Dean J and Ghemawat S (2004) MapReduce: Simplified data processing on large clusters. 6th conference on Symposium on Opearting Systems Design \& Implementation, Seattle, WA, USA

4. Fitzpatrick B (2004) Distributed caching with Memcached. Linux Journal 2004(124):5

5. Vianna $E$ (2011) Modeling performance of the hadoop online prototype. International Symposium on Computer Architecture, Vitoria

6. Xie J (2010) Improving Map Reduce performance through data placement in heterogeneous Hadoop clusters. IEEE International Symposium on Parallel \& Distributed Processing, Atlanta

7. Ishii M, Han J, Makino H (2013) Design and performance evaluation for hadoop clusters on virtualized environment. In: 2013 International Conference on Information Networking (ICOIN), pp. 244-249

8. Chao T, Zhou H, He Y, and Zha L (2009) A Dynamic MapReduce Scheduler for Heterogeneous Workloads. Techical paper online, IEEE Computer Society.

9. Ranger C, Raghuraman R, Penmetsa A, Bradski G, and Kozyrakis C (2007) Evaluating MapReduce for multi-core and multiprocessor systems. HighPerformance Computer Architecture, Proc. IEEE 13th Int'l Symp. High Performance Computer Architecture, Scottsdale, AZ, USA

10. Dean J and Ghemawat S (2004) MapReduce: simplified data processing on large clusters. In Op. Systems Design \& Implementation
11. Hendricks J, Sambasivan RR, and Sinnamohideenand S, Ganger GR (2006) Improving small file performance in object-based storage. Technical report, Carnegie Mellon University Parallel Data Lab, Online technical report

12. Berezecki M, Frachtenberg E, Paleczny M, Steele K (2011) Many-Core KeyValue Store. International Green Computing Conference and Workshops, Orlando, FL, USA

13. Mandal A et al (2011) Provisioning and Evaluating Multi-domain Networked Clouds for Hadoop-based Applications. Third International Conference on Cloud Computing Technology and Science, Athens, Greece

14. Shafer J, Rixner S, Cox AL (2010) The Hadoop distributed filesystem: Balancing portability and performance. IEEE International Symposium on Performance Analysis of Systems \& Software, White Plains, NY, USA

15. Leverich J, Kozyrakis C (2010) On the energy (in)efficient of Hadoop clusters. ACM SIGOPS Operating systems Review, Indianapolis, IN, USA

16. Chun B (2010) An Energy Case for Hybrid Datacenters. ACM SIGOPS Operating System Review, Indianapolis, IN, USA

17. Wang G, Butt A, Pandey P, and Gupta K (2009) Using realistic simulation for performance analysis of MapReduce setups. LSAP. ACM, Munich, Germany

18. Issa J, Figueira S (2010) Graphics Performance Analysis Using Amdahl's Law. IEEE/SCS SPECTS, International Symposium on Performance Evaluation of Computer and Telecommunication System, Ottawa, ON, Canada

19. Issa J, Figueira S (2011) Performance and power-Consumption Analysis of Mobile Internet Devices. IEEE IPCC-International Performance Computing and Communications Conference, Austin, TX, USA

20. Apache Software Foundation: Official apache hadoop website: http:// hadoop.apache.org. (2015)

21. Wiktor T et al (2011) Performance Analysis of Hadoop for Query Processing. International Conference on Advanced Information Networking and Applications, Fukuoka, Japan

22. Ekanayake J, Pallickara S, and Fox G (2008) MapReduce for data intensive scientific analysis. In: Fourth IEEE Intl. Conf. on eScience, pp. 277-284.

23. Chu C-T, Kim SK, Lin Y-A, Yu Y, Bradski G, Ng AY, and Olukotun K (2007) Map-Reduce for machine learning on multicore. NIPS, Vancouver, B.C., Canada, pp. 281-288.

24. Emanuel V (2011) Modeling Performance of the Hadoop online Prototype. ISCA, San Jose, CA, USA

25. Dejun J and Chi GPC (2009) EC2 Performance Analysis for Resource Provisioning of Service-Oriented Applications. International Conference on Service-Oriented Computing, Stockholm, Sweden

26. Ibrahim S, Jin H, Lu L, Qi L, Wu S, and Shi X (2009) Evaluating MapReduce on Virtual Machines: The Hadoop Case. International Conference on Cloud Computing, Bangalore, India

27. Stewart R (2010) Performance and Programmability of High Level Data Parallel Processing Languages. http://www.macs.hw.ac.uk/ rs46/papers/ appt2011/RobertStewart_APPT2011.pdf

28. Tan J, Pan X, Kavulya S, Gandhi R, and Narasimhan P (2008) Salsa: Analyzing Logs as State Machines. In: Workshop on Analysis of System Logs.

29. Tan J, Pan X, Kavulya S, Gandhi R, and Narasimhan P (2009) Mochi: Visual Log-analysis Based Tools for Debugging Hadoop. In: HotCloud.

30. Therdphapiyanak J, Piromsopa K (2013) An analysis of suitable parameters for efficiently applying K-means clustering to large TCPdump data set using Hadoop framework. In: 10th International Conference on Electrical Engineering/Electronics, Computer, Telecommunications and Information Technology

31. Jiang DR, B. Ooi B, Shi L, and Wu S (2010) The performance of MapReduce: an in-depth study. Proceedings of the VLDB Endowment, Online Journal

32. Esteves RM, Pais R, Rong C (2011) K-means Clustering in the Cloud - A Mahout Test. In: IEEE Workshops of International Conference on Advanced Information Networking and Applications (WAINA), vol., no., pp.514-519, 22-25 